\title{
Sexual Dimorphism in the Prenatal Digit Ratio (2D:4D)
}

\author{
Frietson Galis · Clara M. A. Ten Broek • \\ Stefan Van Dongen · Liliane C. D. Wijnaendts
}

Received: 11 January 2008 / Revised: 28 January 2009 / Accepted: 30 January 2009/ Published online: 20 March 2009

(C) The Author(s) 2009. This article is published with open access at Springerlink.com

\begin{abstract}
The second to fourth digit ratio (2D:4D) is smaller in human males than in females and hence this trait is sexually dimorphic. The digit ratio is thought to be established during early prenatal development under the influence of prenatal sex hormones. However, the general assumption of early establishment has hardly been studied. In our study, we analyzed the 2D:4D ratio in 327 deceased human fetuses. We measured digit lengths in 169 male and 158 female fetuses ranging from 14 to 42 weeks old. Our results showed a slight, but significant, sexual dimorphism in the expected direction, i.e., females had, on average, a ratio of 0.924 and males a ratio of 0.916 . There was no significant relationship with the presence or absence of minor and major or single and multiple congenital abnormalities. There was a minimal, but significant difference between digit ratios based on digit lengths including and excluding the non-bony fingertip with the values being strongly correlated $(r=.98)$. The prenatal 2D:4D ratio was lower than has thus far been reported for children and adults both for males and females. The extent of the sexual dimorphism in fetuses was similar to that found for children, but lower than for adults. The 2D:4D ratio, thus, seems to increase after birth in both men and women, with the second digit growing faster than the fourth digit (positive
\end{abstract}

F. Galis $(\bowtie)$. C. M. A. Ten Broek

Institute of Biology, Leiden University, PO Box 9516, 2300 RA Leiden, The Netherlands

e-mail: f.galis@biology.leidenuniv.nl

S. Van Dongen

Evolutionary Ecology Group, University of Antwerp,

Antwerp, Belgium

L. C. D. Wijnaendts

Department of Pathology, Free University Medical Centre,

Amsterdam, The Netherlands allometric growth of digit two) and perhaps more so in women than in men. Therefore, the sexual dimorphism is probably determined by prenatal as well as by postnatal developmental processes.

Keywords 2D:4D · Finger ratios - Prenatal development . Sex differences

\section{Introduction}

In humans, the relationship of the length of the second (index) finger and fourth (ring) finger (2D:4D) is, on average, lower in male than in female adults (e.g., Ecker, 1875; George, 1930; Manning, Scutt, Wilson, \& Lewis-Jones, 1998) and, hence, this ratio shows a sexual dimorphism. There is considerable variation in the $2 \mathrm{D}: 4 \mathrm{D}$ ratio within populations. In both sexes, the second digit can be longer or shorter than the fourth digit (Jones, 1944). It is generally assumed that the sexual dimorphism is established during early prenatal development under the influence of sex hormones and the 2D:4D ratio is usually assumed to be stable after early prenatal stages (e.g., Garn, Burdi, Babler, \& Stinson, 1975; Manning et al., 1998; Phelps, 1952) or after two years of age (Brown, Hines, Fane, \& Breedlove, 2002). In contrast, Trivers, Manning, and Jacobson (2006) found in a longitudinal study of Jamaican children a slight but significant increase in the 2D:4D ratio for boys and girls from 7-13 to 1117 years of age four years later, with a high correlation between measurements on the same individuals. This suggests that the 2D:4D ratio does not remain constant throughout life, but slowly increases in a stable manner during childhood.

These findings are in agreement with an earlier study by Schultz (1926) on Caucasian fetuses. Schultz found that 2D:4D ratios larger than one (second digit longer than fourth 
digit) were very rare in young fetuses and that their incidence increased from $2 \%$ in fetuses of three months old to $14 \%$ in fetuses six to ten months old.

Despite the findings of Schultz (1926) and Trivers et al. (2006), it is generally assumed that the differences in digit length ratios between females and males are already established in utero and Garn et al. (1975) and Phelps (1952) are usually cited to substantiate this claim. However, these studies did not provide evidence for this. Garn et al. (1975) found in 56 human fetuses that the average rankings according to size of the phalanges and metacarpals in fetuses of 7 weeks and older corresponded rather well with those in adults. Interestingly, Garn et al. found no significant differences between male and female fetuses and they combined the data in their results. The conclusion of Garn et al. was rather general in that the relative sizes of bones of the hand were established early during gestation. Phelps (1952) did not study the 2D:4D ratio in fetuses but only in adults and concluded, citing other studies, that in fetuses 2D:4D ratios occur that are smaller than one, larger than one, and equal to one. For this, Phelps cited the above-mentioned study of Schultz (1926), as well as a smaller study by Huizinga (1949). Malas, Dogan, Evcil, and Desdicioglu (2006) found in a study of 161 fetuses without external abnormalities a significantly larger 2D:4D ratios for female fetuses than for male fetuses. The 2D:4D ratios that Malas et al. found were similar to those found in other studies of adults (Table I), suggesting that 2D:4D ratios do not change after birth. The findings of Malas et al. are, thus, in contrast with those of Schultz (1926) and of the longitudinal study of Trivers et al. (2006).

In this study, we analyzed the 2D:4D ratios in 347 deceased fetuses to test whether the 2D:4D ratio is already established during early fetal development. For this purpose, we tested whether there were differences between male and female fetuses, and whether there was a change with age by comparing our data with those in the literature on children and adults. Finally, because our sample was not unbiased, but consisted of deceased fetuses, we tested whether there was a relationship of the $2 \mathrm{D}: 4 \mathrm{D}$ ratio and the presence or absence of minor and major congenital abnormalities to investigate whether comparison with the general population was possible.

\section{Method}

\section{Subjects}

Since 1980, deceased human fetuses which arrive for examination at the VU Medical Centre in Amsterdam are routinely radiographed ventrally and laterally with hands taped in as straight a position as possible $(23 \mathrm{~mA}, 70-90 \mathrm{kV}$, 4-12 s, Agfa Gevaert D7DW Structurix films). The ventral radiographs of 643 fetuses that died between 1992 and 1999 were used for this study. A total of 316 fetuses were excluded for measurements because the fingers could not be properly positioned for the radiographs. In total, 169 male and 158 female fetuses (M, 28 weeks \pm 11 weeks; range, 14-42 weeks) were examined for the left and right hand 2D:4D. For male fetuses, 129 left hands and 138 right hands were measured and, for 98, measurements were made on both hands. For female fetuses, 118 left hands and 130 right hands were measured and, for 90 , both hands could be measured. Data on ethnicity were not available, but the patient population of the VU Medical Centre is predominantly Caucasian. Gestational ages were provided by the gynecologist, based on the time since the first day of the last menstruation (postmenstruation).

\section{Measures}

Measurements of the digits were made from the midpoint of the proximal end of the proximal phalanx bone to the midpoint of the distal end of the distal phalanx bone of the second and fourth digit (Fig. 1) with a transparent ruler with resolution of $.01 \mathrm{~cm}$. For 53 randomly sampled digit ratios, digit lengths were also measured for the complete finger (i.e., including the non-bony finger tip). Measurements were carried out by one of two investigators without prior knowledge of the autopsy reports (however, several congenital

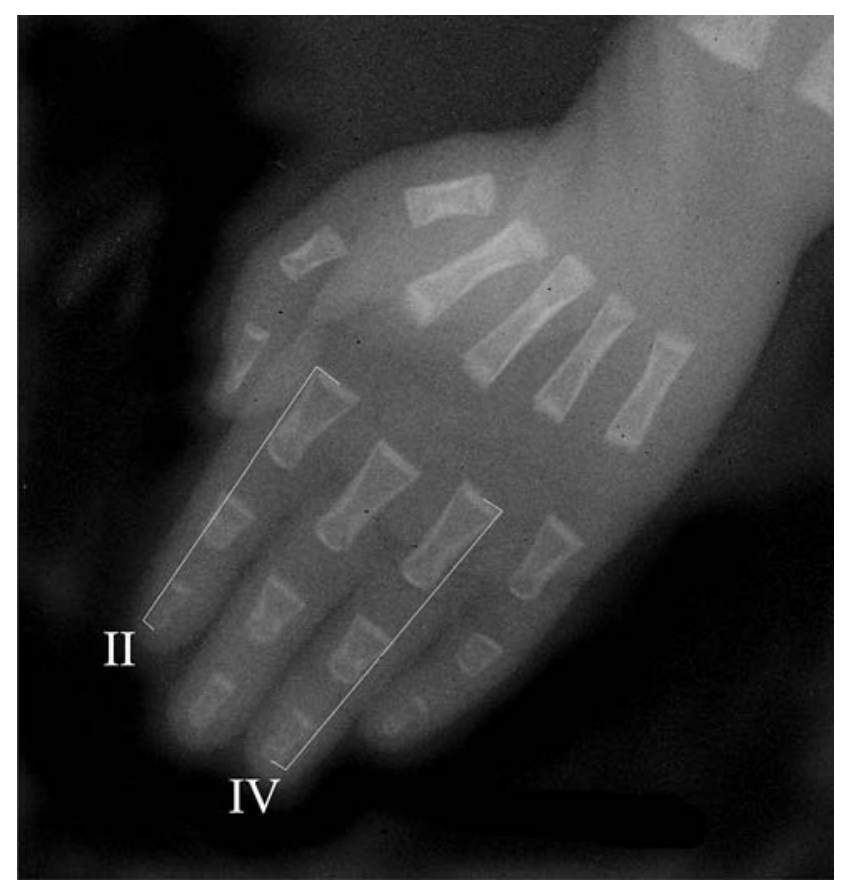

Fig. 1 Radiograph of right hand. Lines indicate length of the midpoint of the proximal end of the proximal phalanx to the midpoint of the distal end of the distal phalanx. The fourth digit is longer than the second digit and this is the case in all but one specimen 
anomalies could be seen in radiographs) and of the sex of the fetus. To compare the accuracy of the measurements of the two investigators, 38 randomly sampled fetuses were independently measured by each investigator. The repeatability of the digit length measurements was high $(r=.99)$. The entire procedure of positioning a fetus and making a radiograph was standardly repeated in the hospital for larger fetuses (for technical reasons) and for 32 randomly sampled fetuses of this set we repeated the measurements. The repeatability of the measurements also was high ( $r=.94$ for the right hand ratio and $r=.95$ for the left hand ratio).

\section{Diagnosis of Abnormalities}

Standard autopsy reports were made by pathologists and filed in a national pathological archive (PALGA). We searched the reports for single and multiple congenital abnormalities. Furthermore, we distinguished between minor and major congenital abnormalities following Merks, van Karnebeek, Caron, and Hennekam (2003) for external and skeletal abnormalities and Lancaster and Pedisich (1995) for further abnormalities. We opted for a classification in terms of minor/ major and single/multiple abnormalities, because it reflects our expectations on the strength of the deleterious effects of these abnormalities. The condition of the environment (e.g., the mother) and the condition of the individual both influence the chance of a premature death. In addition, we considered dysmaturity and embryonal tumors to be major abnormalities and prematurity and the presence of an infection a minor abnormality (for a more detailed description of the abnormalities, see Galis et al., 2006).

\section{Statistical Analysis}

2D:4D ratio was calculated on both hands of each individual. Note that this was not possible in all individuals, so for the 347 fetuses, at least one 2D:4D was available. For each side and sex, more than 100 observations were available. In order to compare digit ratios between males and females and between hands, and to test for an association with age, a linear model with repeated measures error structure was used (Verbeke \& Molenbergs, 2000). Measurements on both hands within a fetus were thus considered as repeated measurements and the error matrix was of the so-called unstructured type. A separate residual variance for each side and one covariance parameter were estimated. Sex, left or right hand, age (continuous covariate), and all two-way and three-way interactions were added as fixed effects. Furthermore, we analyzed whether there was a relationship between 2D:4D development and the presence or absence of congenital abnormalities and whether they were considered to be minor or major. All analyses were performed in SAS version 9.

\section{Results}

To present the results from our analysis graphically, the raw data and model predictions were plotted (Fig. 2). For both male and female fetuses, there were no significant differences between left and right hand 2D:4D, but, as previously reported, male fetuses had a lower 2D:4D (significant sex effect), $F(1,324)=4.26, p=.03$, but not side, $F(1,324)<1$, or a side by sex interaction, $F(1,323)<1$. The mean values were: male fetuses: left hand $=.915(S D=.034)$, right hand $=.916(S D=.037)$; female fetuses: left hand $=.927$ $(S D=.032)$, right hand $=.923(S D=.034)$. There appeared to be a decrease of the 2D:4D ratio with age which differed between the left and right hand (significant age effect), $F(1,324)=4.19, p=.04$, and a side by age interaction, $F(1,323)=4.00, p=.05$. This association appeared to be comparable between male and female fetuses since there was no significant three-way, $F(1,323)<1$, or age by sex interaction, $F(1,323)<1$. In both male and female fetuses, 2D:4D decreased slightly, but significantly, with age in the
Fig. 2 The 2D:4D ratio in deceased male and female human fetuses. Males have, on average, a slightly lower ratio than females for both hands (.91 vs. .92). The ratio slightly, but significantly declines for the left hand, but not for the right hand
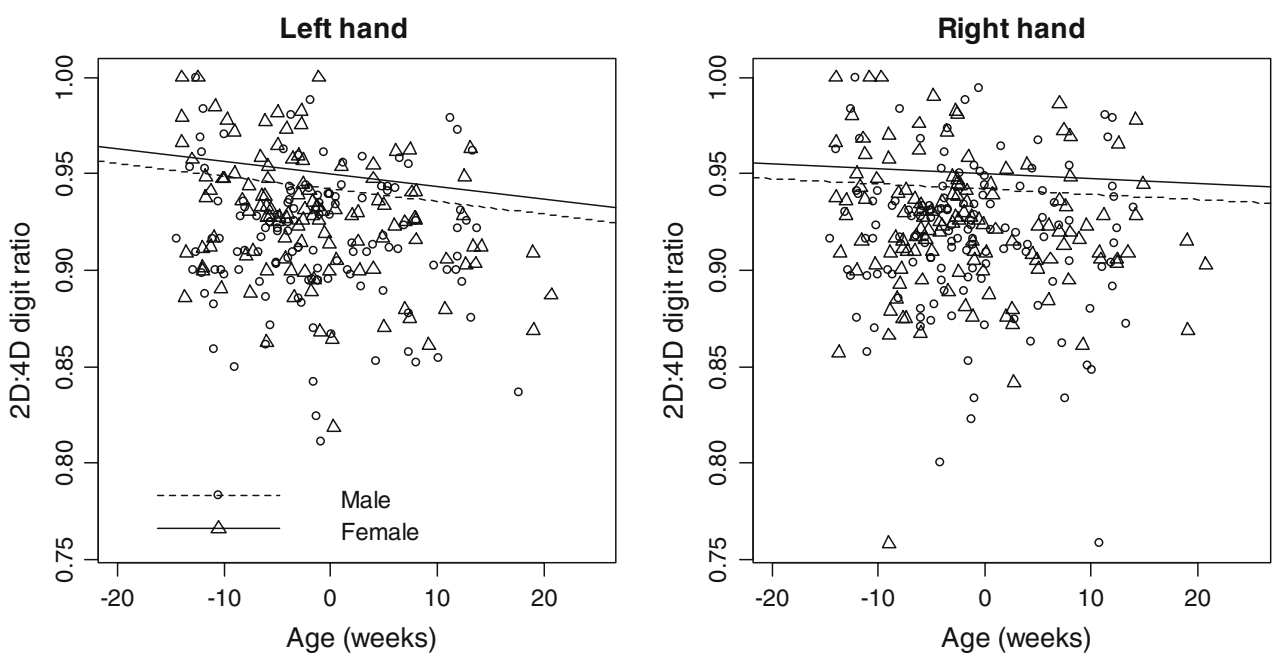
left hand, slope $=-.007$ ( $S E=0.003), p=.008, r^{2}=4.4 \%$, but not in the right hand, slope $=-.003(S E=.003)$, $p=.30, r^{2}=0.6 \%$ (Fig. 2).

Next, we tested whether 2D:4D differed for fetuses with the presence or absence of minor or major congenital abnormalities in a linear model containing the significant effects presented above. The 2D:4D ratios did not differ between fetuses with or without congenital abnormalities, $F(1,303)=2.58, p=.11$. Within fetuses with congenital abnormalities, there was no significant difference in $2 \mathrm{D}: 4 \mathrm{D}$ ratio for minor and major abnormalities, $F(1,303)<1$.

Finally, we tested whether 2D:4D ratios differed when the complete finger, including the non-bony finger tips, was measured. The 2D:4D ratios differed only slightly, although significantly $(.925, S E=.004$ with non-bony finger tip, versus $.921, S E=.004$ without non-bony finger tip), paired $t$-test, $d f=52, p<.0001$. The correlation between the two types of measurements was strong $(r=.97, d f=51$, $p<.0001)$.

\section{Discussion}

Male and female fetuses of 14 weeks gestational age or older showed a small, but significant, difference in 2D:4D ratio. The difference was in the expected direction, i.e., males had a lower ratio than females. The extent of the sexual dimorphism was smaller than found in studies of adults (Table 1). It was comparable in size to that found for children in the studies of Trivers, Manning, Thornhill, Singh, and McGuire (1999) and Trivers et al. (2006), but smaller than in an earlier smaller study on Turkish fetuses without external abnormalities by Malas et al. (2006) (Table 1). Unexpectedly, we did find a very weak negative relationship with gestational age for the left hand, but not for the right hand.

In agreement with what has been speculated in the literature and with earlier data from Malas et al. (2006), our data suggest that already at a gestational age of 14 weeks the sexual dimorphism is present. This is in agreement with the hypothesis that early levels of sexual hormones have a lasting influence on 2D:4D ratios (Brown et al., 2002; McFadden, Westhafer, Pasanen, Carlson, \& Tucker, 2005; Okten, Kalyoncu, \& Yaris, 2002, but see Buck, Williams, Hughes, \& Acerini, 2003).

We measured the length of the digits from radiographs not directly from the fingers and we excluded the non-bony finger tip. Measurement method may make a small difference, as was shown for measurements from photocopies compared to direct measurements from fingers (Manning, Fink, Neave, \& Caswell, 2005). However, these differences are very small compared to the differences between our fetal ratios and those in the literature on children and adults. In addition, we found that the differences between measurements with and without finger tip were minimal and strongly correlated.

The 2D:4D ratios in male and female fetuses in this study were, on average, smaller than the ratios found in children by Manning, Stewart, Wilson, and Lewis-Jones (2004) and Trivers et al. (2006) and again smaller than those found in adults (Manning et al., 1998, 2004; McFadden \& Shubel, 2002; Putz, Gaulin, Sporter, \& McBurney, 2004). In agreement with the findings of the longitudinal study of Trivers et al. (2006), this suggests that after birth 2D:4D ratios may increase. We found that in all cases the fourth digit was larger than the second digit, except in one case where the length of the digits was equal, whereas in adults, especially in females, the second digit is regularly longer than the fourth. Schultz (1926) also rarely found 2D:4D ratios $>1$ in fetuses of three months old $(2 \%, n=200)$ whereas in larger fetuses he found a higher incidence (14-18\%, 4-9 months old, $n=526)$ and in adults a yet higher incidence $23 \%(n=200)$. Similarly, Mierzecki (1946) reported that digit ratios were lower in Caucasian and Black fetuses than in adults (2D:4D $<1$ in 0 $16.3 \%$, N not given, no data given on adults). However, Malas et al. (2006) found in a study of 161 fetuses without

Table 1 2D:4D ratios for males and females of varying age in different studies

\begin{tabular}{|c|c|c|c|c|c|}
\hline Study & Age & $\begin{array}{l}\text { Male 2D:4D } \\
\text { (right, left) }\end{array}$ & $\begin{array}{l}\text { Female 2D:4D } \\
\text { (right, left) }\end{array}$ & $\begin{array}{l}\text { Number } \\
\text { of subjects }\end{array}$ & $\begin{array}{l}\text { Measurement } \\
\text { type }\end{array}$ \\
\hline Manning et al. (1998) & $2-25$ years & $.98, .98$ & $1.00,1.00$ & 800,20 per year & Direct \\
\hline Saino, Leoni, and Romano (2006) & $21-30$ years & $.966, .967$ & $.978, .975$ & 138 males, 154 females & Digital scans \\
\hline Putz et al. (2004) & $18-30$ years & $.949, .955$ & $.974, .979$ & 213 males, 120 females & Photocopies \\
\hline Russell (2006) & 20 years & $.953, .951$ & $.977, .976$ & 53 males, 77 females & Photocopies \\
\hline Trivers et al. (2006) & $7-13$ years & $.932, .924$ & $.944, .938$ & 54 males, 54 females & Photocopies \\
\hline $\begin{array}{l}\text { Trivers et al. (2006) (same } \\
\text { subjects } 4 \text { year later) }\end{array}$ & $11-17$ years & $.944, .934$ & $.951, .955$ & 54 males, 54 females & Photocopies \\
\hline Manning et al. (2004) & $6-14$ years & .941 & .951 & 263 males, 265 females & Photocopies \\
\hline Malas et al. (2006) & $10-40$ weeks & .945 & 1.006 & 83 males, 78 females & Direct \\
\hline This study & 14-42 weeks & $.916, .916$ & $.923, .927$ & 169 males, 158 females & Radiographs \\
\hline
\end{tabular}


external abnormalities digit ratios that were comparable to those of adults in other studies (Table 1) and Huizinga (1949) found in a study on 100 Caucasian fetuses that they had more often a 2D:4D ratio $>1(65.0 \%, 3-9$ months old $)$ than children $(45.7 \%, 4-15$ yrs old, $n=1750)$ and adolescents and adults $(38.3 \%,>15 \mathrm{yrs}$ old, $n=562)$. Huizinga's study also differed from our study and the one of Malas et al. (2006) in that he did not find a difference between male and female fetuses (64.3\% for male and $65.9 \%$ for female fetuses); however his sample size was small. In the older studies of Schultz (1926), Mierzecki (1946), and Huizinga (1949) no digits ratios were given, only whether ratios were larger than one, smaller than one or equal to one.

Hence, the studies of Trivers et al. (2006), Schultz (1926), Mierzecki (1946), and our study in comparison with studies on children and adults suggest that after birth the digit ratio increases, which would imply a positive allometric (i.e., relatively stronger) growth of the second digit after birth, but this is not supported by the study of Huizinga (1949) and Malas et al. (2006).

Several studies have found a significant positive relationship between 2D:4D ratio and medical problems (Down syndrome, ADHD) (McFadden et al., 2005; Putz et al., 2004; Schneider, Pickel, \& Stalla, 2006, but see Buck et al., 2003). In contrast to this, a significant negative relationship has been found between the 2D:4D ratio and physical prowess (Putz et al., 2004). In our study, we did not find a significant relationship between 2D:4D ratio and the presence or absence of minor or major congenital abnormalities.

Our results were necessarily based on a select sample of fetuses, i.e., deceased fetuses, because it is not yet possible to carry out such studies on healthy fetuses. It is possible that our results were biased because of this. However, the cause of death can be maternal (e.g., cervix insufficiency) as well as fetal and not all fetuses had abnormalities. We did not find a relationship between the $2 \mathrm{D}: 4 \mathrm{D}$ ratio and the presence or absence of minor or major abnormalities and this suggests that the bias was probably limited.

We conclude that female fetuses have, on average, a higher 2D:4D ratio than male ones. Hence, the sexual dimorphism in 2D:4D ratio appears to be already present by 14 weeks of gestation. Comparison with data on children and adults in the literature reveals that the $2 \mathrm{D}: 4 \mathrm{D}$ ratio may increase after birth in both men and women and slightly more so in women than in men. This would imply that the second digit grows relatively faster than the fourth one after birth, especially in women. Furthermore, this would imply that both prenatal and postnatal developmental processes are involved in the determination of the difference in 2D:4D ratio between males and females. This hypothesis is supported by the majority of other studies, including the largest one (Schultz, 1926), but not by some smaller studies. Furthermore, we found only a minimal difference between measurements of digits with and without the non-bony fingertip. Finally, we did not find a relationship between the 2D:4D ratio and the presence or absence of abnormalities and the severity of abnormalities.

Acknowledgements We thank Richard Wassersug for proposing to us to study the 2D:4D ratio in this group of human fetuses. We thank Jaap van Veldhuisen and Ron Otsen of the photography division of the Institute of Pathology of the Free University Medical Centre (VUMC) for high quality radiographs and the photographs of Fig. 1.

Open Access This article is distributed under the terms of the Creative Commons Attribution Noncommercial License which permits any noncommercial use, distribution, and reproduction in any medium, provided the original author(s) and source are credited.

\section{References}

Brown, W. M., Hines, M., Fane, B. A., \& Breedlove, S. M. (2002). Masculinized finger length patterns in human males and females with congenital adrenal hyperplasia. Hormones and Behavior, 42, 380-386.

Buck, J. J., Williams, R. M., Hughes, I. A., \& Acerini, C. L. (2003). In utero androgen exposure and 2nd to 4th digit length ratiocomparisons between healthy control and females with classical congenital adrenal hyperplasia. Human Reproduction, 18, 976-979.

Ecker, A. (1875). Some remarks about a varying character in the hands of human. Archiv für Anthropologie, 8, 68-74.

Galis, F., Van Dooren, T. J. M., Feuth, H., Ruinard, S., Witkam, A., Steigenga, M. J., et al. (2006). Extreme selection in humans against homeotic transformations of cervical vertebrae. Evolution, 60, 2643-2654.

Garn, S. M., Burdi, A. R., Babler, W. J., \& Stinson, S. (1975). Early prenatal attainment of adult metacarpal-phalangeal rankings and proportions. American Journal of Physical Anthropology, 43, 327332.

George, R. (1930). Human finger types. The Anatomical Record, 46, 199-204.

Huizinga, J. (1949). The digital formula in relation to age, sex and constitutional type. Proceedings Koninklijke Nederlandse Akademie van Wetenschappen, 52, 403-411; 587-593.

Jones, F. W. (1944). Principles of anatomy as seen in the hand. London: Williams and Wilkins.

Lancaster, P., \& Pedisich, E. (1995). Congenital malformations, Australia 1981-1992. Sydney: Australian Institute of Health and Welfare, National Perinatal Statistics Unit.

Malas, M. A., Dogan, S., Evcil, E. H., \& Desdicioglu, K. (2006). Fetal development of the hand, digits and digits ratio (2D:4D). Early Human Development, 82, 469-475.

Manning, J. T., Fink, B., Neave, N., \& Caswell, N. (2005). Photocopies yield lower digit ratios (2D:4D) than direct finger measurements. Archives of Sexual Behavior, 34, 329-333.

Manning, J. T., Scutt, D., Wilson, J., \& Lewis-Jones, D. I. (1998). The ratio of 2 nd to 4 th digit length, a predictor of sperm numbers and concentrations of testosterone, luteinizing hormone and oestrogen. Human Reproduction, 13, 3000-3004.

Manning, J. T., Stewart, A., Wilson, J., \& Lewis-Jones, D. I. (2004). Sex and ethnic differences in 2nd to 4th digit ratio of children. Early Human Development, 80, 161-168.

McFadden, D., \& Shubel, E. (2002). Relative lengths of fingers and toes in human males and females. Hormones and Behavior, 42, 492500 . 
McFadden, D., Westhafer, J. G., Pasanen, E. G., Carlson, C. L., \& Tucker, D. M. (2005). Physiological evidence of hypermasculinization in boys with the inattentive type of attention-deficit/ hyperactivity disorder (ADHD). Clinical Neuroscience Research, $5,233-245$.

Merks, J. H. M., van Karnebeek, C. D., Caron, H. N., \& Hennekam, R. C. (2003). Phenotypic abnormalities: Terminology and classification. American Journal of Medical Genetics, 123A, 211-230.

Mierzecki, H. (1946). Over de morphologie der hand. Ciba-tijdschrift, $18,573-575$.

Okten, A., Kalyoncu, M., \& Yaris, N. (2002). The ratio of second- and fourth-digit lengths and congenital adrenal hyperplasia due to 21hydroxylase deficiency. Early Human Development, 70, 47-54.

Phelps, V. R. (1952). Relative index finger length as a sex-influenced trait in man. American Journal of Human Genetics, 4, 72-89.

Putz, D. A., Gaulin, S. J. C., Sporter, R. J., \& McBurney, D. H. (2004). Sex hormones and finger length: What does 2D:4D indicate? Evolution and Human Behavior, 25, 182-199.

Russell, D. C. (2006). Raise your hand if you think you are attractive. Personality and Individual Differences, 40, 997-1005.
Saino, N., Leoni, B., \& Romano, M. (2006). Human digit ratios depend on birth order and sex of older siblings and predict maternal fecundity. Behavioural Ecology and Sociobiology, 60, 34-45.

Schneider, H. J., Pickel, J., \& Stalla, G. K. (2006). Typical female 2nd4th finger length (2D:4D) ratios in male-to-female transsexualspossible implications for prenatal androgen exposure. Psychoneuroendocrinology, 31, 265-269.

Schultz, A. H. (1926). Fetal growth of man and other primates. Quarterly Review of Biology, 1, 465-521.

Trivers, R., Manning, J., \& Jacobson, A. (2006). A longitudinal study of digit ratio (2D:4D) and other finger ratios in Jamaican children. Hormones and Behavior, 49, 150-156.

Trivers, R., Manning, J. T., Thornhill, R., Singh, D., \& McGuire, M. (1999). Jamaican Symmetry Project: Long-term study of fluctuating asymmetry in rural Jamaican children. Human Biology, 71, 417-430.

Verbeke, G., \& Molenbergs, G. (2000). Linear mixed models for longitudinal data. New York: Springer Verlag. 